\title{
The Slip Induction Task: Creating a window into cognitive control failures
}

\author{
Amanda J. Clark • Rupa Parakh • Daniel Smilek • \\ Eric A. Roy
}

Published online: 13 October 2011

(C) Psychonomic Society, Inc. 2011

\begin{abstract}
Regardless of age, education, or social status, we all experience moments where we ask ourselves, "what was I thinking?!?" Typically, we experience errors of this sort, or slips of action, during routine tasks that require a whole sequence of movements. However, most action slip research has focused on creating response conflict within single movements. The Slip Induction Task (SIT) is a step toward creating response conflict within truer to life action routines. As such, the SIT attempts to induce action slips in a well-learned movement routine through the occasional presentation of unexpected cues. We find that the SIT is able to reliably induce action slips, especially when the unexpected cue necessitates a movement to an unexpected target location. Furthermore, when participants are able to avoid an action slip, these changes in movement routine are accompanied by a cost in speed.
\end{abstract}

Keywords Attention - Action slip - Cognitive control · Attention failure

\section{Introduction}

In almost every hour of our lives, humans are able to easily perform a number of complex activities simply because those tasks have become highly practiced and routine.

\footnotetext{
A. J. Clark · R. Parakh • D. Smilek • E. A. Roy

University of Waterloo,

Waterloo, Canada

\author{
A. J. Clark $(\square)$ \\ Rotman Research Institute, \\ Baycrest, 3560 Bathurst Street, \\ Toronto, Ontario, Canada M6A 2E1 \\ e-mail: aclark@rotman-baycrest.on.ca
}

Consider, for example, your first time sitting in front of the steering wheel of a car. At the time, every aspect of driving required a keen sense of attention, and often mistakes were made as a result of pure inexperience. With a few years of exposure to driving a vehicle, however, it is amazing to reflect on how seamlessly all of the required actions are coordinated. Unfortunately, though, errors of this sort are still frequent and certainly not unique to driving. Perhaps you walk into the kitchen and forget what you went there to do, or you fail to add sugar to your coffee due to an interruption. Indeed, these errors permeate almost every simple activity that we perform at one point or another.

Slips of action are frustrating, distracting, costly, and, at times, physically dangerous (Robertson, 2003). A hallmark of action slips is that they are characterized as errors that occur during routine, well-known tasks. As such, these are not the types of errors that a person makes while a novice at a task; instead, actions slips occur in highly routine actions, where the person is quite skilled at the task but, for one reason or another, his or her actual behavior deviates from his or her intention (Heckhausen \& Beckmann, 1990; Schwartz, 1995). These deviations from intention seem to be especially prevalent at points in time when conscious attention to the routine task at hand is absent, disengaged, or insufficient (Robertson, Manly, Andrade, Baddeley, \& Yiend, 1997).

Although interest in this area of attention, action control, and action slips began with William James (1890), who suggested that "habit diminishes the conscious attention with which our acts are performed," James's contemporaries have developed a research career around investigating the types of errors that occur during habitual tasks. Indeed, Reason and colleagues have extensively studied action slips by using subjective reports from participant diaries. These diary studies have revealed that action slips are unintended, 
automatically triggered action sequences that are particularly apt to occur when highly practiced tasks are performed in familiar environments (Jonsdottir, Adolfsdottir, Cortez, Gunnarsdottir, \& Gustafsdottir, 2007; Reason, 1977, 1979, 1984; Reason \& Mycielska, 1982). Furthermore, slips of action appear to be highly related to one's experience with the task at hand and frequently occur at points in time where a decision must be made about how to proceed with the subsequent steps of the routine task. In fact, Reason (1979) theorized that it was at these junctures or decision points that participants had to access information about the goals of the tasks and what actions had been done up to that point to achieve those goals. Broadbent, Cooper, FitzGerald, and Parkes (1982) have expanded theoretically upon this finding to suggest that familiar tasks and familiar environments make very few demands on our conscious attention and, as such, resources are available to think about or do a number of other things, which leaves us vulnerable to distractions, boredom, and slips.

Norman and Shallice $(1986,2000)$ and Shallice and Burgess (1993) have complemented Reason's research by suggesting their model of action, which implicates the existence of two independent systems that allow for the cognitive control of actions. The first, the contention scheduling system (CS), directs well-learned actions, operates mainly outside of conscious control, and therefore requires very few resources. Conversely, the supervisory attention system (SAS) requires consciously controlled attention to allow for the execution of novel or dangerous tasks. Inherent in Norman and Shallice's model is the assumption that for accurate action production and efficient attention control, the use of these systems must be balanced and that, at optimal times, a switch between them may be required, as when the goals of a task change.

Taken together, Reason's theories and Norman and Shallice's model of action suggest that slips of action likely result from a failure to adequately monitor and interrupt the functioning of the CS when required. However, other researchers have indicated alternative explanations for the experience of action slips, such as degraded task context (Botvinick \& Bylsma, 2005), dual-task conditions (Giovanetti, Schwartz, \& Buxbaum, 2007; Giovannetti et al., 2010; Humphreys, Forde, \& Francis, 2000), the presence of external, environmental distractors (Buxbaum, Schwartz, \& Montgomery, 1998; Giovannetti et al., 2010), time pressure (Betsch, Haberstroh, Molter, \& Glockner, 2004), and daytime sleepiness (Wallace, Vodanovich, \& Restino, 2003). These explanations and Norman and Shallice's model of action are not necessarily mutually exclusive, however. Instead, elements such as degraded task context, dual-task conditions, and external distractions may explain mismatches between intentions and actual behavior, or in other words, slips of action, insofar as they explain why the SAS does not become deployed to interrupt the CS when necessary.

Likewise, recent theories that focus on the neural substrates of cognitive control appear not to discount a theory involving a SAS and CS but, instead, provide additional detail with respect to these processes. The bulk of this work within cognitive neuroscience has indicated an involvement of areas within the prefrontal cortex (PFC) and the parietal cortex, as well as subcortical areas-specifically, the basal ganglia and the anterior cingulate (ACC), which work together to achieve accurate goal-directed behavior. Given that cognitive control involves handling the demands of changing circumstances and internal goals, it is clear that monitoring the performance of routine tasks requires not just the detection of errors, but also the maintenance of task-relevant information, detection of conflict, and inhibition of inappropriate behavior as well (Fassbender et al., 2004).

Clinical and neuroimaging evidence points to the PFC as being important for representing and maintaining taskrelevant information, as well as suppressing irrelevant information that might compete or distract from the task goals (Braver, Barch, Gray, Molfese, \& Snyder, 2001; Carter et al., 1998; Casey, Tottenham, \& Fossella, 2002; Kok, Ridderinkhof, \& Ullsperger, 2006; Miller \& Cohen, 2001). Furthermore, Miller and Cohen specifically posited that the PFC achieves this by biasing activation toward goal-relevant acts and away from goal-irrelevant acts, even when the irrelevant pathway to action is stronger. In other words, when possible responses are in competition with each other, the PFC biases activation in other parts of the brain toward the response that is in line with the task goals. As such, according to Miller and Cohen's theory, when a well-practiced routine response (strong pathway and likely controlled by the CS) becomes task irrelevant or incorrect and conflicts with another response (weaker pathway) due to a change in the task, the PFC must act as a "switch operator" (Miller \& Cohen, 2001, p. 184) by biasing activation toward the weaker pathway. This therefore switches control from the stronger CS pathway to the weaker, more novel pathway that requires SAS control.

Similarly, Corbetta and Shulman (2002) also supplemented Norman and Shallice's model by proposing two fronto-parietal networks that control the allocation of attention. The first, more dorsal and goal directed, involves the superior frontal cortex and intraparietal cortex, and the other, more ventral and stimulus directed, involves the inferior frontal cortex and temporoparietal cortex. According to Corbetta and Shulman's evidence, these two networks work mostly independently, the dorsal route operating on the basis of expectancy, like the CS, and the ventral route continually monitoring for task-relevant stimuli and acting as an alerting system when appropriate. 
Importantly, though, their theory suggests that these two networks must interact upon the detection of unexpected, task-relevant information. Indeed, the expected pathway or circuit must be broken via a subnetwork within the ventral frontoparietal network that directs control away from the more dorsal route to action and, instead, toward the more consciously controlled SAS, which controls actions in a more stimulus-directed fashion (Corbetta \& Shulman, 2002). In addition to the fronto-parietal networks that have been associated with cognitive control, additional recruitment of the presupplementary motor area (Jonides, Badre, Curtis, Thompson-Schill, \& Smith, 2002; Sharp et al., 2010), as well as subcortical and midline structures such as the basal ganglia (Aron et al., 2007; Casey et al., 2002; Chambers et al., 2006; Fassbender et al., 2004; Kok et al., 2006) and the ACC (Botvinick, Cohen, \& Carter, 2004; Fassbender et al., 2004; Jonides et al., 2002), have been associated with both detecting conflict and inhibiting expected motor output, as well as general outcome monitoring and the detection of erroneous actions.

These data surrounding the neural correlates of cognitive control have primarily elicited errors using varieties of the Eriksen flanker task (Eriksen \& Eriksen, 1974), in which movements are slower and more erroneous when conflict/ incongruency exists between the target and its flankers; the Simon task (Simon \& Berbaum, 1990), in which movements are slower and more erroneous when the cue and target are incongruent; the continuous performance task (Paxton, Barch, Racine, \& Braver, 2008; Rosvold, Mirsky, Sarason, Bransome, \& Beck, 1956); go/no-go tasks, in which selective attention is required for an infrequent stimulus; and the stop signal task (Logan, Cowan, \& Davis, 1984), where occasional stop signals indicate the required inhibition of a routine response. All of these tasks, however, provide an overly simplified view of the cognitive control of everyday action, one in which participants are required to choose from one of only a couple of responses, where each movement is completed in isolation from the last and where participants rarely develop a learned, expected response. To more closely approximate the occurrence of action slips in actual daily routines, we believe that it is important to better capture the complexity of many daily tasks, tasks that almost always require a whole sequence of actions to complete and are often completed on the basis of routine. Consequently, the focus of this study is to examine the extent to which the previously mentioned theories of cognitive control, which suggest dual attention control systems with fronto-parietal networks, explain the errors that occur when an action is occasionally altered within a complex sequence of routine actions. This additional layer of complexity is a feature of the SIT, which is novel as compared with other, previously mentioned tasks, which elicit errors due to response conflict.

To accomplish this goal, the Slip Induction Task (SIT) was developed to induce slips of action by requiring occasional deviations from a well-learned sequence of actions to target buttons. In a laboratory environment, the SIT attempts to elicit action slips by first teaching participants a series of hand movements to target buttons through the use of spatially compatible arrow cues. Following this learning phase, where presumably a routine action plan has been established, participants proceed to the alteration phase of the task, in which the arrow cue for one of the movements within the sequence violates the learned routine. This alteration to the base sequence may involve the cue's appearing in an unexpected spatial location only (positional alteration; see Fig. 1), pointing toward an unexpected target button only (directional alteration; see Fig. 2), or both being in a spatially unexpected location and pointing to an unexpected target button (combined alteration; see Fig. 3). These alterations to the routine sequence were designed to directly assess whether simple distracting information (positional alteration) and/or movement goal changes (both directional and combined alterations) might affect the induction of action slips.

In Experiment 1, we measured a participant's accuracy in following the instructions of the arrow cue, even when it
Fig. 1 Depiction of the cue for a movement with no alteration compared with the cue for a movement with a positional alteration (correct target button is highlighted)
No Alteration (Cue as Expected)

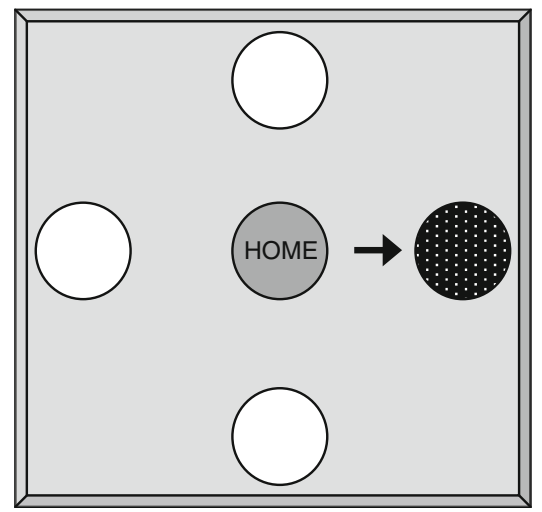

Example of a Positional Alteration

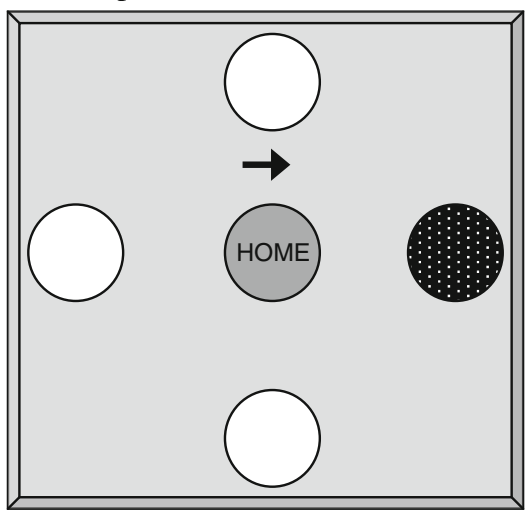


Fig. 2 Depiction of the cue for a movement with no alteration compared with the cue for a movement with a directional alteration (correct target button is highlighted)
No Alteration (Cue as Expected)

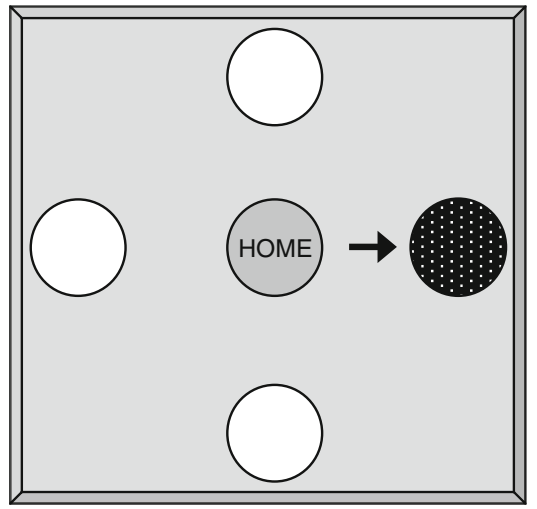

Example of a Directional Alteration

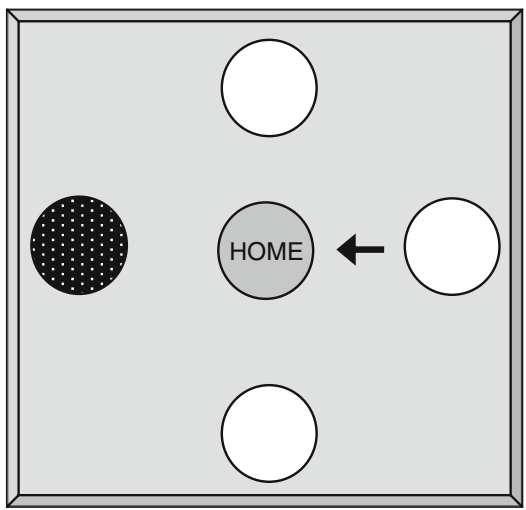

was pointing to an unexpected target or when located in an unexpected spatial location. We expected that experience with the sequence during the learning phase would cause it to become highly routine and, accordingly, would involve the recruitment of Norman and Shallice's (1986, 2000) and, presumably, the dorsal fronto-parietal brain network of cognitive control (Corbetta \& Shulman, 2002). Furthermore, we expected that participants would continue to rely on this more efficient system during the alteration phase of the experiment. Therefore, we predicted that use of CS attentional control would become problematic for performance whenever participants encountered an altered trial. We expected this to be particularly true when such an alteration required a change in the expected action plan or movement goal (a directional or combined alteration; see Figs. 2 and 3, respectively). Such a situation would require adequate monitoring of task-relevant stimuli via the ventral fronto-parietal network (Corbetta \& Shulman, 2002) and, likely, the involvement of the PFC to bias activation away from the expected movement and, instead, to the new required target (Miller \& Cohen, 2001)or in other words, a switch to the SAS to inhibit the expected or routine action plan.

As we have seen, though, the intervention of the SAS is not always dependable, especially when conscious control of attention is lacking, as is typical in routine actions
(Norman \& Shallice, 1986, 2000). Consequently, we predicted that a number of errors would be made in all three alteration conditions. Importantly, though, we expected that not all types of alterations would induce equal numbers of errors. Instead, while we expected the largest decrement in accuracy whenever a change in movement goal was required, we predicted the most errors in situations where the unexpected arrow cue was located in the expected spatial location (directional alteration; see Fig. 2), since the unexpected arrow would be spatially compatible with the expected movement goal. This was based on the hypothesis that the arrow itself would draw attention toward the expected target button and, as such, would result in a reduced likelihood that participants would notice the unexpected endogenous information contained in the arrow cue.

Although two of the three types of alterations in the slip induction procedure (directional and combined alterations; see Figs. 2 and 3, respectively) require changes in the movement goal, the other type of alteration (positional) simply involves incorporating a cue that is spatially incongruent with the desired target button. This was accomplished by positioning an arrow cue that was pointing to the expected movement goal, in an unexpected, and incongruent, spatial location. This type of manipulation
Fig. 3 Depiction of the cue for a movement with no alteration compared with the cue for a movement with a combined alteration (correct target button is highlighted)
No Alteration (Cue as Expected)

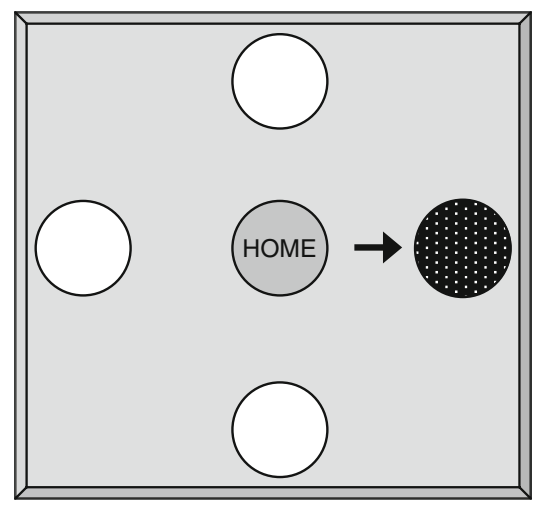

Example of a Combined Alteration

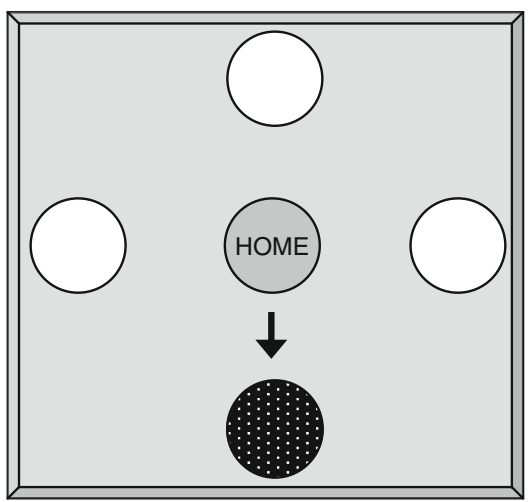


made it important for the participant to ignore the exogenous, potentially distracting spatial information. We predicted that this type of alteration would induce action slips because of the Simon effect (Simon \& Berbaum, 1990). Simon reported that participants took longer to respond to a target that was incompatible with its cue, because they had to override their natural inclination to move toward the spatial location of the cue. As such, even though such a movement might not be part of the expected sequence, we hypothesized that participants might naturally move to a target that was near the cue, even when the cue continued to point to the expected target button.

In our second experiment, we expanded upon our investigation not only by examining accuracy in more detail, but also by looking at the time required to complete a sequence of movements and the microstructure of the sequence timing. As such, the same SIT procedure was adopted, but in this experiment, timing measures, such as sequence time (ST), response initiation time (IT), movement time (MT), and time to return to the home location (RHT) were also incorporated. These measures were included to allow for the investigation of how participants responded temporally before, during, and after trials that were altered.

As has already been established, the CS is a more efficient use of resources and is used when executing welllearned action routines. This system however is subject to failure, since it allocates less conscious attention to the task at hand. Using the SART, Robertson and colleagues (1997) have demonstrated that when an inhibition failure is made on their task, response times for the movement just prior to the error are faster than on trials immediately prior to a correctly inhibited trial. Presumably, this maps onto the SIT such that just before an alteration occurs, participants may be prone to error if they are using the CS and are preparing the expected movements before the appearance of the actual cue. Given this, we predicted that movements would be faster on trials directly preceding an erroneous trial. What was unclear, however, was whether the cause of this particular effect was with respect to ITs or MTs. Moreover, we also predicted that on altered trials in which the correct movement was made, longer ITs and MTs would be required to allow for inhibiting the expected plan and programming a new movement plan.

Furthermore, in real-life action production, the only way to guard against slips of action is to learn from them when they do occur. In keeping with this, it is expected that participants will attempt to avoid action slips whenever possible. Therefore, consistent with findings of strategic posterror slowing (Gratton, Coles, \& Donchin, 1992; Rabbitt \& Rodgers, 1977), we predicted that following an alteration that resulted in an error, participants would attempt to prevent subsequent errors by slowing down their movements and focusing more closely on the action sequence. Con- versely, however, following an altered yet correctly executed trial, we expected that participants' timing measures would not differ from those associated with nonaltered trials.

Finally, Experiment 2 was also designed to determine whether participants' propensity to make errors on the SIT would correlate with other measures of cognitive control. We expected a positive correlation between performance on the SART (Robertson et al., 1997) and the SIT, since both tasks require the inhibition of a well-learned, routine response. In addition, we also predicted that participants who reported experiencing more attention failures in daily life via the Attention Related Cognitive Errors Scale (ARCES; Cheyne, Carriere, \& Smilek, 2006) would also make not only more errors on the SART, but also more errors on altered trials in the SIT.

\section{Experiment 1}

Method

\section{Participants}

Twenty-four right-handed undergraduate students (12 of them male) were recruited from the paid participant pool at the University of Waterloo to take part in this experiment, where slips of action were induced within the SIT. All participants were between the ages of 18 and 25 years and were right-handed.

\section{Stimuli and apparatus}

In this experiment, participants moved to target buttons as instructed by arrows cues that appeared on a computer screen. The sequence of arrow stimuli used in this experiment was created using Micro Experiments Laboratory. Each of the arrow cues that were displayed using this program measured $70 \mathrm{~mm}$ in length (creating a visual angle of between $11^{\circ}$ and $16^{\circ}$ ) and $50 \mathrm{~mm}$ in height (creating a visual angle of between $9^{\circ}$ and $13^{\circ}$ ) and were displayed $125 \mathrm{~mm}$ from the center of the screen in one of the four directions. The sequence of arrow stimuli was shown on a 15-in. flat-screen monitor that was inverted to allow the stimuli to be projected onto a mirror that occluded the participants' hands (Fig. 4). Situated under the mirror was a $16 \times 16$ in. button board equipped with five 2-in. diameter buttons, one located centrally with the others located to the north, south, east, and west of the central home button.

\section{Design and procedure}

Upon arrival at the laboratory, participants were informed of the general procedures of the study, including the risks 


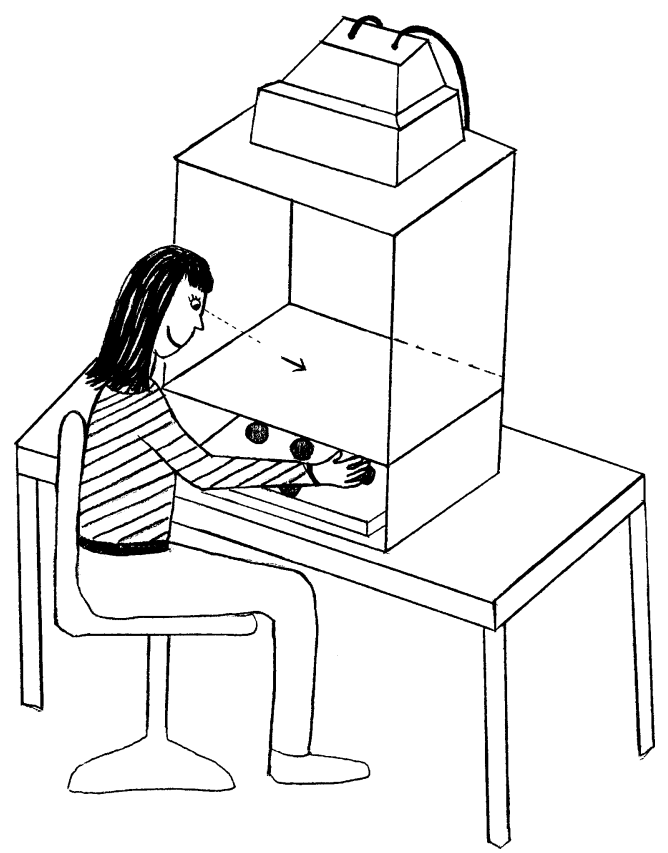

Fig. 4 Depiction of SIT apparatus

and benefits that they might incur. This discussion included making sure that they knew that the study would require them to come into the lab on two separate days. On the first day of testing, participants were randomly assigned to practice a sequence of seven hand movements to target buttons for one, three, or six blocks of 120 trials; this constituted the learning phase of the experiment. Regardless of the amount of practice trials, however, the learning phase always began with participants quickly getting acquainted with the button locations on the response board. Once familiar with the response board and the button locations, participants were informed that a series of arrows were going to appear and that their task was to move as quickly and as accurately as possible to the buttons on the response board that corresponded with those arrows.

The sequence contained a total of seven movements to four target buttons located around a central home button. For each movement in the sequence, a directional arrow appeared above, below, to the right, or to the left of the central button, and it pointed to the spatially congruent target button. As such, for each movement, participants received both compatible exogenous (the physical location of the arrow on the screen) and endogenous (the pointed direction of the arrowhead) cuing information about the target location. During the learning phase, the position and direction of the arrow cues were never manipulated, and the participants were informed that this was the case.

Five to 9 days following the completion of this learning phase, participants began their second session of the experiment by becoming reacquainted with the movement sequence through rehearsing it for an additional 60 trials. Subsequently, participants entered the alteration phase of the experiment, where they continued to execute the same sequence of movements for an additional five blocks of 120 trials. However, in $28 \%$ of these 600 sequences, one of the seven arrow cues was altered by changing the spatial location of the cue, by changing the direction of the arrowhead and, therefore, the actual movement goal, or by changing both of these components. As such, three types of alterations to the learned movement sequence were introduced.

Type I Positional alterations (Fig. 1) For this type of alteration, the goal of the movement remained as practiced in the sequence; however, the spatial position of the arrow cue was changed. As such, for positional alterations, when participants expected to see an arrow located to the right, indicating a movement to the right target for their third movement, they actually saw an arrow pointed to the right but located above, below, or to the left of the central home button for that movement in the sequence. Therefore, the only unexpected aspect of the cue was the exogenous information that was communicated by the spatial positioning of the arrow cue.

Type II Directional alterations (Fig. 2) Directional alterations involved the spatial position of the arrow cue being as practiced, but the actual direction of the arrowhead was changed, and therefore, the goal of the movement was changed from what was expected. This meant that when participants expected to see an arrow located to the right, indicating a movement to the right target for their third movement, they actually saw an arrow pointed up, down, or to the left but still located to the right of the central home button. Consequently, this type of alteration manipulated only the endogenous information that the participants received.

Type III Combined alterations (Fig. 3) In the case of a combined alteration, both the endogenous and exogenous information that the participant received was changed. Thus, both the spatial location of the arrow cue and the pointed direction of the arrowhead were randomly changed from what the participants expected at that point in the sequence. As such, for combined alterations, even though participants may have expected an arrow to appear to the right and point to the right target for the third movement, the arrow cue could have actually appeared in any one of the other spatial locations and was pointed to any one of the other targets.

In this alteration phase of the experiment, seventy of the sequences contained a directional alteration: Forty-two were altered positionally and another 28 involved the combined 
alteration. Before beginning the alteration phase, all participants were informed that a portion of the sequences would be changed in some way, and it was stressed that their task would be to follow the arrow's instructions. As such, if an arrow appeared that pointed to a new target, they were to move to that new target as quickly and as accurately as possible.

Turning now to the sequence of events for each trial (in both the learning and alteration phases), a fixation cross appeared in the center of the screen at the beginning of each sequence. This fixation cross remained for between 500 and $1,500 \mathrm{~ms}$ to ensure that participants were not able to predict when the sequence of arrow cues was going to begin. Once the fixation cross disappeared, the participant pressed the central home button, which automatically triggered the onset of the first arrow cue. Upon seeing this arrow cue, the participants released the home button and quickly moved to the target to which it pointed. Once reaching the target, participants quickly pressed the button, released it, and immediately returned to the central home button. The base sequence of movements that was used in this experiment was right, down, up, down, right, left, then up.

\section{Analyses}

The analyses for this experiment were divided into two groups: those for the learning phase and those for the alteration phase. To determine the extent to which the participants acquired the movement sequence during the learning phase, we compared participants' accuracy, as well as their average time required to complete an entire sequence (ST), using one-way ANOVAs.

For the alteration phase of the experiment, number of errors made by each participant was tallied and grouped according to whether the error was on a directionally altered trial, a positionally altered trial, a trial with a combined alteration, or a trial that was not altered in any way. These four error frequencies were then converted into percentage accuracy scores by dividing the number of errors made on each type of trial by the total number of possible errors that could have been made. The resulting accuracy scores were tested against each other by using an omnibus mixed model ANOVA in which the percentage accuracies for the four types of possible errors (unaltered, positional, directional, and combined) were included as within-subjects factors and amount of practice during the learning phase (one, three, or six blocks of 120 trials) was included as a between-subjects factor. This analysis was used to determine whether any or all of the alteration types used in the SIT were successful in provoking action slips. Where appropriate, Tukey's HSD post hoc tests, with an alpha level of .05 , were also used to determine significance.
Results and discussion

Learning phase Before looking specifically at the ability of our task to induce slips of action, it is important to examine participant performance during the learning phase. As such, the results discussed in this section are grouped according to the amount of practice trials that each participant completed. Amount of practice during the learning phase did not significantly impact accuracy during the practice trials, $F(2,21)=1.61, p=.223$. Since the groups did not differ in their accuracy as a result of the number of practice trials completed, it may suggest that participants reached ceiling accuracy within the first block of trials and maintained this accuracy throughout subsequent training blocks.

With respect to ST, however (Fig. 5), even though between-group analyses revealed that the average ST for the first block of 120 practice trials did not differ, $F(2,23)=$ $0.372, p=.693$, there was a main effect of practice group on average time to complete the final block of 120 trials (trials 241-360 for those that competed three blocks of training and trials 601-720 for those that completed six blocks of training), $F(2,21)=10.66, p=.001$. Furthermore, post hoc analyses using Tukey's HSD approach revealed that average ST for those who completed only one block of 120 practice trials $(M=388.4 .52, S D=742.38)$ was significantly longer than the average ST of the final 120 practice trials for those who completed three $(M=2924.24, S D=545.49)$ or six blocks $(M=2629.61, S D=327.07 ; \mathrm{p}<.05)$. However, the average ST for the final 120 practice trials for those who completed three blocks did not differ from that for those who completed six blocks $(p>.05)$.

Alteration phase Upon examining accuracy in response to the different types of alterations, a main effect of alteration type was found, $F(1,23)=94.24, p<.001$. Furthermore, Tukey's HSD post hoc analyses for this main effect

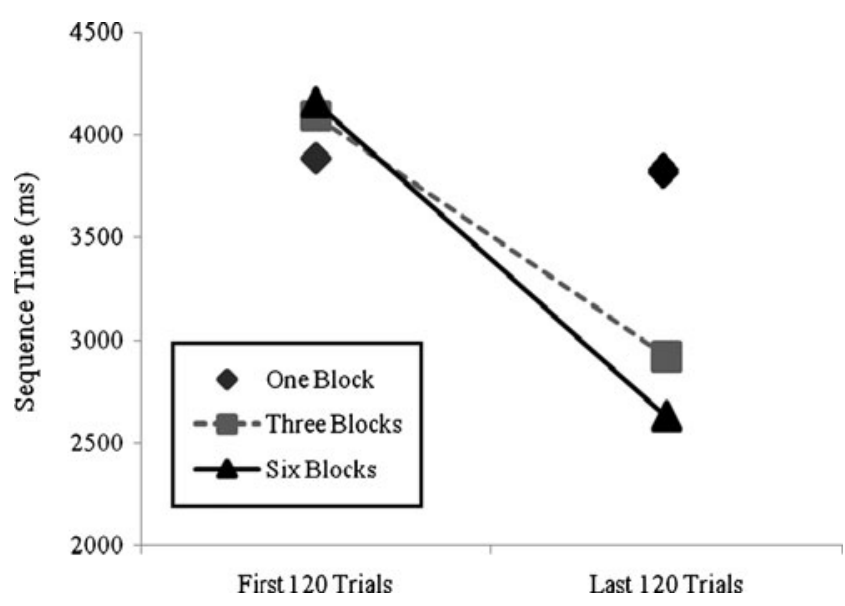

Fig. 5 Mean sequence time (in milliseconds) during practice phase: Experiment 1 
indicated that unaltered trials were completed more accurately $(M=99.1, S D=2.1)$ than trials that contained a positional $(M=91.0, S D=14.1)$, directional $(M=13.3, S D=$ $16.5)$, or combined $(M=39.8, S D=24.1 ; p<.05)$ alteration (see Fig. 6) and that accuracy for all three types of alterations also differed from each other $(p<.05)$.

This indicates that, as was predicted, participants had difficulty when they encountered unexpected cue information. Furthermore, accuracy was worst when the unexpected cue information required an unexpected movement and was also located adjacent to the expected target button (directional alteration). This likely suggests that the exogenous information contained in the altered arrow cue actually acted as an attractor toward the expected yet inappropriate target. In other words, these results indicate that inhibition of the expected movement was most difficult when the new (unexpected) movement was cued from the expected location.

Additionally, in terms of our hypothesis regarding the Simon effect (Simon \& Berbaum, 1990), while trials containing a positional alteration rarely resulted in an error, Tukey's HSD post hoc analyses confirmed that these trials were still performed less accurately than unaltered trials $(p<.05)$. This means that even when the movement goal remained as expected, an incompatibility between the locations of the arrow cue and the expected target button was sometimes sufficient to induce an error. However, practice amount did not significantly impact accuracy during the alteration phase $(p>.05)$, nor did it interact with alteration type $(p>.05)$. Given these results, this experiment demonstrates that manipulations to the spatial location and pointed direction of the expected arrow cues, as was used in this procedure, are sufficient to induce action slips but that the range of practice that was selected was not sufficient to create practice-related differences in accuracy.

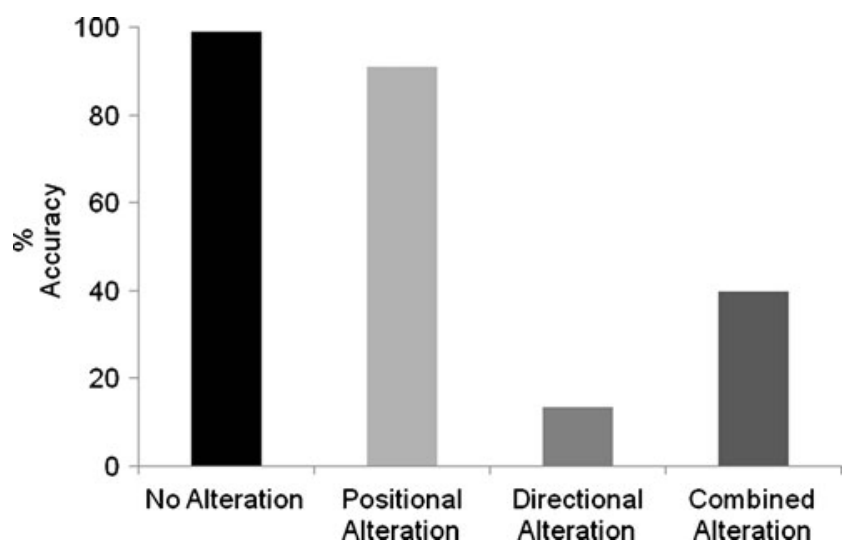

Fig. 6 Accuracy on unaltered trials compared with trials with a positional, directional, or combined alteration: Experiment 1

\section{Experiment 2}

The purpose of this experiment was to replicate and build upon the findings of Experiment 1 by looking at participants' overall accuracy on altered trials, as well as by examining the timing of the actual movements. We were interested in learning more about the actual timing of an SIT-induced action slip to determine whether these errors have any relationship with the speed of sequence completion (ST) and, furthermore, whether measures of response IT, MT, and/or RHT were able to shed light on how these errors occur from a temporal standpoint. As such, the basic SIT procedure used in Experiment 1 was adopted in Experiment 2, and the above-mentioned timing measures were also collected and analyzed. Moreover, in an effort to determine the degree to which the attentional-controlrelated errors from the SIT are congruent with other attention-related errors, participants in Experiment 2 also completed the SART and the ARCES, and correlations between these measures are discussed.

\section{Method}

\section{Participants}

Thirty right-handed undergraduate students (12 of them male) between 18 and 25 years of age were the participants in this experiment.

\section{Design and procedure}

The stimuli, apparatus, procedures, and most of the analyses were identical to those used in Experiment 1. Additionally, timing measures were also collected. To break down how the individual timing measures were isolated within the SIT, be reminded that at the beginning of each trial, a fixation cross appeared, and the participant was told to press the central home button upon the disappearance of that fixation cross, which automatically triggered the onset of the first arrow cue. Upon seeing this arrow cue, the participant released the home button, and it was at the release of the home button that the participant's IT was recorded; this also triggered the beginning of the MT measure. Once the target was reached, participants pressed the button that signaled the end of MT. Finally, the time that elapsed between the release of the target button and the next depression of the home button was also recorded and is referred to as the RHT.

Participants in Experiment 2 also completed the SART and the ARCES. Half of the participants were randomly assigned to complete these tasks before beginning the learning phase of the SIT, while the other half completed the tasks after finishing the alteration phase of the SIT. The 
SART was completed as per the parameters outlined by Robertson and colleagues (1997). As such, 25 targets, the digit 3, were quasirandomly interspersed with 200 additional digits, 1 through 9. All digits were presented for $250 \mathrm{~ms}$ and were followed by a mask with a 900-ms duration. Participants were instructed to press a key on the keyboard each time a digit appeared, except when that digit was 3. As such, participants were asked to inhibit their response to an infrequent target. They were encouraged to complete this task as quickly and as accurately as possible. Participants were also asked to complete the ARCES, a 12item self-report questionnaire of one's frequency of attention failures in everyday life (see the Appendix for a copy of the ARCES).

\section{Analyses}

Similar to those conducted in Experiment 1, the analyses for this experiment were divided into two groups: those for the learning phase and those for the alteration phase. For the learning phase, a one-way ANOVA was used to compare the participants' average ST for the last 120 trials of practice, and a Tukey's HSD test with an alpha level of .05 was used to determine whether each group significantly differed from each other. For the alteration phase, participant errors were tallied on the basis of whether the error was on a directionally altered trial, a positionally altered trial, a trial with a combined alteration, or a trial that was not altered in any way. These were subsequently converted into percentage accuracy scores. In addition to simply considering which alteration types impacted accuracy, we were also interested in learning whether more experience with the sequence during the learning phase would impact participants' subsequent accuracy during the alteration phase. As such, an omnibus mixed model ANOVA was conducted in which the percentage accuracies for the four types of possible errors (unaltered, positional, directional, and combined) were included as within-subjects factors and amount of practice during the learning phase (one, three, or six blocks of 120 trials) was included as a between-subjects factor. In addition, simple planned contrasts were integrated in this analysis such that accuracy on trials that were not altered was compared with accuracy on positionally altered trials, directionally altered trials, and trials that contained a combined alteration. Subsequently, three additional $t$-tests (with corresponding Bonferoni $\alpha$-level corrections) were required to directly compare accuracy between positionally altered trials, directionally altered trials, and trials with a combined alteration.

Also, in light of our hypotheses about how one's timing of movements before, during, and after an altered trial might influence performance, three groups of statistical tests were conducted. The first group of tests compared participants' ITs, MTs, and RHTs of unaltered movements, as compared with actual altered movements that were executed correctly or erroneously. The second group of tests looked at participants' timing for the movements that occurred immediately before a correct or erroneous altered movement. The third set of tests explored the same measures for the movements immediately following an error or a correctly completed altered movement. For each of these mixed model ANOVAs, while the dependent measure of interest changed (IT, MT, or RHT), practice group was always used as a between-subjects factor, and the within-subjects factor was always whether the altered movement was completed correctly or erroneously.

To determine the extent to which other measures of inattention would predict performance on the SIT, a number of regression analyses were computed. The SART task features measures of hits (situations where a buttonpress is withheld accurately), commission errors (situations where a buttonpress is not withheld when it should be), and omission errors or false alarms (situations where a buttonpress is withheld inappropriately). In addition, participants' subjective reports of experiencing attention failures in daily life were scored on the ARCES questionnaire. Using a stepwise regression to eliminate the potential effect of practice amount, each of these components, SART commission errors, SART omission errors, and ARCES score were regressed upon the total number of SIT errors, the number of SIT errors made on altered trials, and the number of SIT errors made on unaltered trials. Finally, to ensure that these results were interpretable in light of the literature available on these two measures, correlations were also conducted to investigate the degree to which ARCES scores predicted commission errors on the SART.

Results

\section{Learning phase}

Before looking specifically at the ability of our paradigm to induce slips of action, it is important to examine participant performance during the training session. As such, the results discussed in this section are grouped according to the amount of training that each participant received. Considering participants' average ST for their final block of practice trials (the last data point for each group in Fig. 7), it was found that those with additional training were able to execute the sequence more quickly than were those with less training. This trend was confirmed statistically through a between-groups ANOVA, which showed a main effect of practice amount on ST, $F(2,27)=14.402, p<.001$. In addition, Tukey's HSD post hoc analyses of this main effect revealed that while participants with one block of training $(M=4,250.34, S D=906.14)$ were significantly 


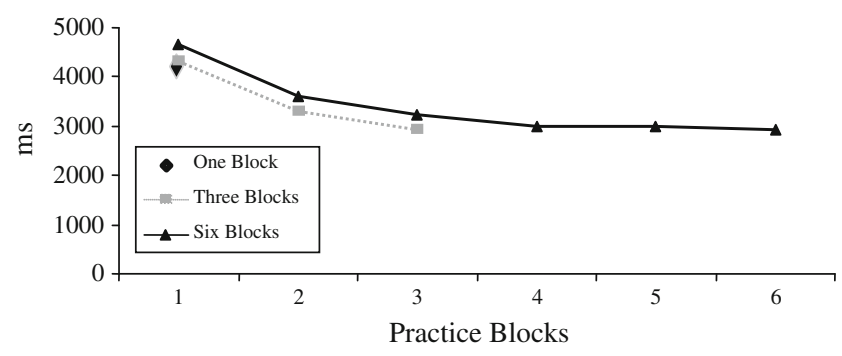

Fig. 7 Mean ST (in milliseconds) across blocks of practice: Experiment 2

slower than those with three $(M=2,934.44, S D=269.61)$ or six $(M=2,919.79, S D=569.98)$ blocks of training $(p<.05)$, those groups with more training (groups 3 and 6) were not significantly different from each other $(p>.05)$.

\section{Accuracy during alteration phase}

Also consistent with the findings from Experiment 1, a main effect of alteration type on accuracy, $F(3,81)=$ 178.39, $p<.001$, was found (see Fig. 8). However, no main effect of practice amount during the learning phase was observed, $F(1,27)=0.972, p=.395$. Furthermore, no interaction was seen between these variables of practice amount and alteration type, $F(6,81)=0.610, p=.722$.

Considering the main effect of alteration type, however (see Fig. 6), a series of planned simple contrasts and $t$-tests confirmed that, even after considering an adjusted critical $p$ value of .0125 (a Bonferoni correction was used to account for making multiple comparisons), each alteration type significantly differed from each other. Planned simple contrasts revealed that unaltered trials were completed significantly more accurately than were trials with directional alterations, $F(1,27)=598.94, p<.001$, positional alterations, $F(1,27)=17.09, p<.001$, and combined alterations, $F(1,27)=212.28, p<.001$. In addition, while accuracy was significantly worse in response to a combined alteration than to a positional alteration, $t(29)=10.56, p<$

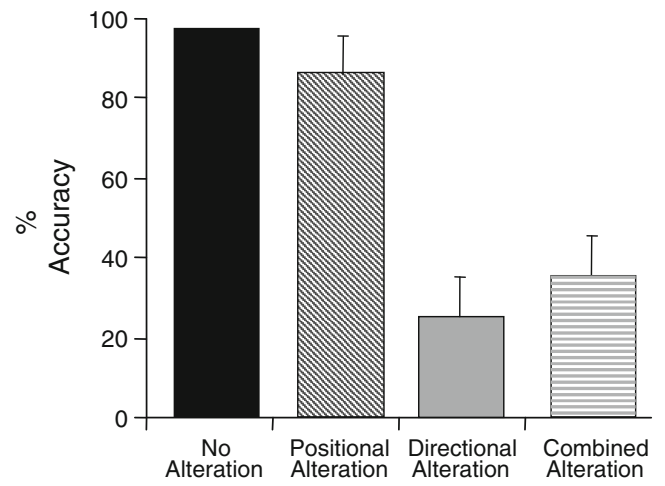

Fig. 8 Accuracy on unaltered trials compared with trials with a positional, directional, or combined alteration: Experiment 2
.001, accuracy after a directional alteration was also lower than for both combined and positional alteration types, $t(29)=$ $3.57, p=.001$, and $t(29)=13.89, p<.001$, respectively.

\section{The timing microstructure of trials}

In this section, ST will be broken down into its microstructure, and as such, the individual measures of IT, MT, and RHT will be examined. These results are described in Table 1. All timing comparisons are considered according to whether the alteration resulted in an error or a correctly executed movement.

\section{Altered movements}

On the basis of the data presented in Table 1, it is clear that participants are slower on trials on which an alteration occurs. While the main effect of accuracy on IT, $F(1,27)=$ 7.08, $p=.013$, and RHT, $F(1,27)=23.56, p<.001$, suggest that participants are slower to initiate and return to the home position after a movement to a correct target, it appears that this slowing is primarily driven by a dramatic increase in the MTs associated with trials that were executed correctly, as compared with trials that resulted in errors, $F(1,27)=146.09, p<.001$ (see Fig. 9). However, none of these main effects of accuracy on IT, MT, or RHT are accompanied by any effect of practice group, $F(2,27)=0.18, p=.83 ; F(2,27)=0.13, p=.88$; and $F(2,27)=0.70, p=.50$, respectively, nor does any interaction between these variables on accuracy and practice exist, $F(2,27)=1.31, p=.29 ; F(2,27)=0.13, p=.36$; and $F(2,27)=0.48, p=.63$, respectively.

\section{Movements following an alteration}

In addition, the observed effect of accuracy on actual altered movements appears to continue to impact the subsequent movement. However, on the movement following an alteration, Fig. 10 illustrates that following an erroneously completed trial, participants initiated their movement more slowly, as evidenced by a main effect of accuracy on IT, $F(1,27)=23.23, p<.001$, and they also gave themselves more time to prepare for a subsequent move [main effect of accuracy on RHT, $F(1,27)=$ $55.38, p<.001]$. However, no main effect of accuracy for MT, $F(1,27)=0.32, p=.58$, was found.

This finding appears to suggest that participants were sufficiently motivated to prevent further errors by slowing at least some aspects of their performance. Similarly, it may also indicate that additional time, reflected in IT and RHT for the movement following an error, was required to process the action slip and subsequently "reset" the automatized sequence. 
Table 1 Average ST, IT, MT, and RHT (in milliseconds) for unaltered trials, altered trials, and trials that preceded and followed an alteration

\begin{tabular}{|c|c|c|c|c|c|c|c|}
\hline & \multirow[b]{2}{*}{ No Alteration } & \multicolumn{2}{|c|}{ Altered Trials } & \multicolumn{2}{|c|}{ Preceding an Alteration } & \multicolumn{2}{|c|}{ Following an Alteration } \\
\hline & & Error & Correct & Error & Correct & Error & Correct \\
\hline ST & $3,179.30$ & $3,620.85$ & $3,937.94$ & $3,620.85$ & $3,937.94$ & $3,620.85$ & $3,937.94$ \\
\hline IT & 118.79 & 113.11 & 124.75 & 116.75 & 119.95 & 142.66 & 128.05 \\
\hline MT & 184.83 & 192.37 & 441.62 & 171.54 & 191.3 & 317.43 & 312.87 \\
\hline RHT & 175.65 & 164.66 & 182.89 & 173.79 & 185.82 & 506.14 & 319.20 \\
\hline
\end{tabular}

Also, as was observed for actual altered movements, there was no main effect of practice on any of these dependent measures [IT, $F(2,27)=0.18, p=.84$; MT, $F(2,27)=$ $0.11, p=.90$; RHT, $F(2,27)=1.62, p=.22]$, and no interactions between the variables of accuracy and practice exist [IT, $F(2,27)=0.30, p=.75$; MT, $F(2,27)=1.09, p=$ .35 ; RHT, $F(2,27)=2.97, p=.07]$.

\section{Congruence with other measures of cognitive control}

A final goal of Experiment 2 was to examine how errors on the SIT are related to other widely used measures of attention failures - mainly, the SART and the ARCES. We first confirmed a positive relationship between the SART and ARCES $(r=.19, p=.03)$, then subsequently performed a series of stepwise regressions to examine the relationship of each of these measures with the SIT. Importantly, even when using practice amount as the first step in the regression model, this factor did not explain a significant portion of the variance.

The main measures that are discussed with respect to the SART task are total number of commission errors (where a response was made on a no-go trial), total number of omission errors (where a response was withheld on a go trial), and response time (Cheyne, Solman, Carriere, \& Smilek, 2009). Each of these measures was examined in relation to the number of slips made on altered and unaltered trials in the SIT. As was expected, the number of errors that participants made on altered trials did significantly predict the number of commission errors made on the SART task $(r=.55, p=$ .002). Furthermore, the strength of relationship did depend on the type of alteration that induced that error, since

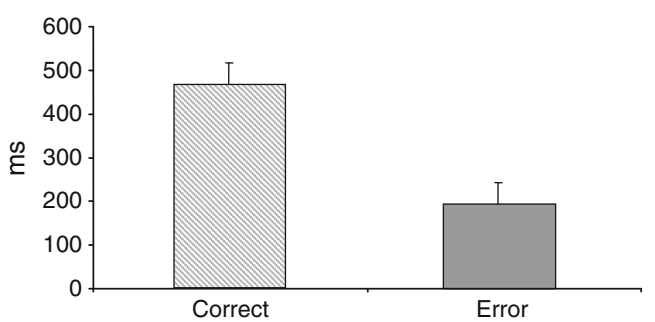

Fig. 9 Average MTs (in milliseconds) for correct or erroneous altered movements accuracy on trials with a directional alteration was more strongly correlated with SART commission errors than was accuracy for trials with a combined or positional alteration. This finding, however, was not observed when looking at the participants' tendency to make omission errors on the SART $(r=.21, p=.27)$.

Upon examining the relationship between the ARCES and the number of slips made in the SIT (on either altered or unaltered trials), regression analyses revealed that it was only the number of errors made when a SIT trial was not altered that predicted a participant's ARCES score $(r=.49, p=.006)$.

\section{Discussion}

One of the initial goals of this study was to replicate the results found in Experiment 1, where the alterations used in the SIT were found to be successful in generating action slips. This follow-up experiment also found evidence that supports this claim. In fact, the results of this study again suggest that while directional alterations were most detrimental to performance, combined and even positional alterations also resulted in significantly more slips than did trials that were not altered in any way.

\section{The microstructure of sequence timing}

The most significant addition made in this experiment was that each trial within each sequence was broken down into its individual behavioral timing measures. As a result, we were able to consider not only STs, but also the individual

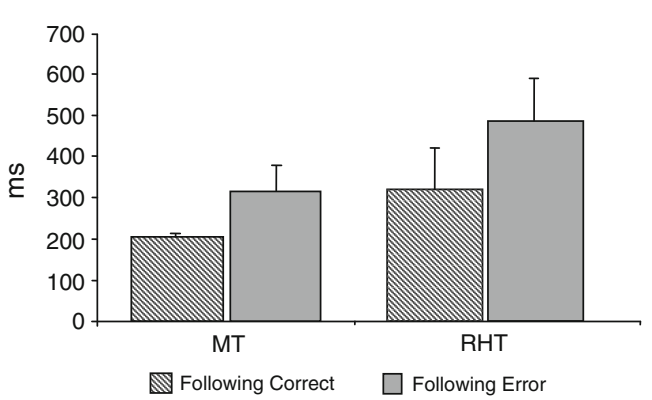

Fig. 10 MTs and RHTs for movements following a correct or erroneous altered movement 
ITs, MTs, and RHTs for each movement in the experiment. This produced an abundance of data that have been categorized according to whether the trial was altered, came before an altered trial, followed an altered trial, or was not closely associated with an alteration. Within this section, those trials that were altered and trials that preceded and followed an alteration will be discussed.

\section{Altered trials}

For trials that were altered, ITs, MTs, and RHTs were examined, and some interesting observations were made. First, as was expected, when participants made an error, they initiated that movement more quickly, and this likely indicates that they were executing a preplanned movement to the expected target, not a movement that was planned to coincide with the actual cue. However, participants moved to the erroneous target in the same amount of time as they did on trials that were not altered.

Conversely, when a participant was able to complete an altered trial correctly, it appears that they made an online correction to their expected movement plan, since their MTs for trials that were executed correctly were significantly lengthened. This may indicate that, even when the correct target button was pressed, participants committed a microslip wherein a movement was initiated toward the expected target location, but that movement was self-corrected. Consequently, it appears that for trials where participants were able to adjust their expected movement plan in response to a change in the sequence, the successful intervention of the SAS occurred during the period of time when they were moving from the home button to the target. Taken together, these results suggest a relationship between speed and errors such that when a routine task is completed quickly, more errors are likely to result, partly because of faster initiation times and also because of a decreased ability to self-correct an erroneous movement during the MT phase.

\section{Trials preceding an alteration}

We hypothesized that movements in the SIT would be highly routinized and, therefore, participants would come to expect movements at particular points in the sequence and would prepare by programming those movements in advance. In doing this, participants should have almost entirely programmed an upcoming movement even before the actual arrow cue appeared, thereby making it difficult for the SAS to interrupt when necessary. In keeping with this hypothesis, we predicted that faster timing before an alteration would allow less time for a CS interruption and, therefore, more errors. However, this hypothesis was not supported by these data. That being said, it is clear that when a participant initiated a movement on an altered trial more quickly, and likely in advance of fully processing the cue information, errors were made more frequently. Considering this, it is possible that the lack of the hypothesized effect resulted from incomplete measurement of the participants' movement planning and programming of the preceding move.

\section{Trials following an alteration}

Now considering the trials that immediately followed an alteration, it does appear that when an error was committed, participants were aware of that error, and both initiated and returned from the subsequent movement more slowly. Therefore, participants do show a posterror slowing effect (Gratton et al., 1992; Rabbitt \& Rodgers, 1977); however, this slowing was not evidenced during the actual MT phase of the movement. Taken together, these results suggest that participants may have required additional time following an error to "reset" the automatized sequence. This is evident both in the increase in time recruited to plan the next move (IT) as well as in a seeming hesitation to move back to the home button to begin the subsequent movement (RHT) in an effort to prevent further errors. What remains unclear however is how long this effect continued. This information would have large practical implications outside the laboratory, since methods for preventing action slips might hinge greatly on knowing how long the impact of making an error, and thereby movement slowing, might help prevent subsequent mistakes.

\section{Congruence with other measures of cognitive control}

One of the potential disadvantages of any experimental task is the fact that events as they occur in the laboratory are never perfect replications of behavior in real-life situations. Because of this, much of the research in the field of action slips has remained within the domain of subjective reporting. Studies of this kind are often extremely rich with descriptive information; however, these descriptions are rarely trusted whole-heartedly. In reaction to this, we attempted to devise an objective method of investigating action slips while also considering previously established methods of looking at attention and control failures.

In an attempt to examine the relationship between these methods and the SIT, all participants in this study completed both the SART and the ARCES questionnaire either at the beginning of the training session or at the end of the experimental session. Before making any claims about how these two tasks relate to the slip induction paradigm, we first verified that the ARCES questionnaire was positively correlated with the number of errors on the SART to the degree that was previously reported in the literature. Since these two measures were related to each other as would be predicted, we proceeded to examine their correspondence with the SIT. 
These analyses showed that SART commission errors on no-go trials - or in other words, trials where a response was made when it should have been inhibited-were strongly correlated with the number of errors that participants made on the SIT. As such, it appears that both of these measures were successful in generating errors, and both appear to tap into one's ability to inhibit a learned action routine.

In addition, the number of slips made on unaltered trials in the SIT was found to significantly correlate with ARCES scores. As such, participants who made more errors on trials that were not altered in any way were also more likely to report attention-related failures, like losing the thread of a conversation, in daily life. This relationship is extremely interesting since it suggests that participants who are more prone to errors in everyday routine tasks are also more prone to making errors in an expected, routine action sequenceeven when no changes to that sequence are required.

\section{General discussion}

The SIT was developed to tax the interplay between Norman and Shallice's $(1986,2000)$ SAS (novel tasks) and CS (welllearned tasks) attention control systems. By generating situations in which the SAS would likely fail to interrupt the "auto-pilot"-like CS, through the use of alterations to the routine sequence, the SIT is able to provide further insight into the mechanisms of an action slip. Previous studies on this topic have been limited by a number of factors that include a reliance on self-report measures such as diaries and questionnaires. This is a concern because, even though self-report measures are rich with descriptive information, they also have disadvantages, which include a reliance on a potentially fallible memory of an event (Baranowski, 1988), the potential for biased reporting based on context and social desirability (Shadish, Cook, \& Campbell, 2002), and the fact that the simple act of self-monitoring modifies actual behavior (Kanfer, 1970). Consequently, these particular experiments were designed to supplement previous work in the field by creating an experimental paradigm in which several slips could be induced in a brief period of time and detailed behavioral timing data could be collected within the laboratory.

The results of these experiments show that the alterations used in the SIT were successful in challenging the relationship between the SAS and CS, since participants made significantly more errors when they encountered unexpected cue information than when the routine movement sequence was not altered in any way. Somewhat surprisingly, however, the degree to which participants practiced the routine sequence did not significantly impact their propensity to make errors. We had hypothesized that increased exposure to the SIT (in the form of practice trials in the learning phase of the task) should have caused the movement sequence to become more routine and, therefore, participants should have increasingly relied on the CS for controlling attention and action. We hypothesized that this increased reliance on the CS would, in turn, lead to a decreased likelihood for CS interruption by the SAS and more action slips when alterations to the expected movement sequence were encountered. However, our results do not support this. One possible explanation for this lack of effect is that the SIT is a fairly unexplored task and that, during the design phase, it was not clear what range of practice trials might be appropriate. In other words, perhaps the sequence was already sufficiently routine to elicit numerous slips even for participants who completed only 120 trials of practice in the learning phase, or alternatively, perhaps 720 trials of practice in the learning phase was not sufficient to elicit the expected training effect. If this is the case, a future study that includes a group of participants who receive significantly fewer or significantly more practice trials would be enlightening.

Another plausible explanation is that within these experiments, by the time participants were at the end of the 600 trials in the alteration phase, even those in the lowpractice condition (one block of 120 trials) had rehearsed the sequence 720 times. It is quite possible that this basically rendered the sequence very well learned even for the low-practice group and dramatically weakened any opportunity for finding an effect of practice on accuracy. To determine the validity of such a hypothesis, a future study should be completed where a much larger sample of participants would complete the SIT where fewer sequences during the alteration phase would be required. In such a design, the alteration phase would consist of only 25 trials, and 6 of those trials would contain an altered arrow cue. With this approach, the integrity of the task would be maintained such that $24 \%$ of the trials would be altered, but the complication of too much additional experience with the task during the alteration phase might be avoided.

Even though participants with additional experience with the sequence during the learning phase did not commit more action slips, all participants did make errors when they encountered alterations to the routine. Furthermore, while all three types of alterations induced slips, they did so to different degrees. In fact, a pattern of accuracy, where positional alterations induced the fewest errors and directional alterations induced the most, was found in Experiment 1 and was replicated in Experiment 2. Given this stable pattern, the individual impact of each of these alteration types is certainly of interest.

Positional alterations were unique in that the unexpected aspect of the cue was simply its spatial location and a stimulus-response incompatibility was created, similar to that which is seen in the Simon task. As such, for accurate performance, participants maintained their expected movement goal, and maintenance of CS attentional control was actually beneficial for accuracy. Furthermore, the fact that the 
positionally altered cues did not appear in a physical location along the expected movement path, which would have created additional competition between movement plans according to Tipper, Lortie, and Baylis's (1992) theory of action-centered attention, was likely also a factor in preventing action slips on these trials. Consequently, errors that were made as a result of a positional alteration were possible only when one became distracted by the cue's stimulus-response incompatibility, likely due to attention being sufficiently biased toward the unexpected exogenous information. It was only under these circumstances that the expected movement plan, and likely the CS, was interrupted.

Considering this marked reduction in the likelihood of making errors on positionally altered trials, it is clear that there is something about directional and combined alterations that increased participants' propensity for action slips. Unlike positional alterations, both directional and combined alterations required a change in the participants' movement goal, and therefore, an incompatibility existed between the expected movement direction and the cue information. As such, a switch from the "auto-pilot" CS was required, and it appears that this necessity significantly impacted participants' performance. This was especially the case with respect to directionally altered cues, which always occurred in a spatial location that was adjacent to the expected target for that point in the sequence. Given that this is the one feature that distinguished it from combined alterations, it follows that there may be something about a directionally altered cue's sharing the physical location of the expected cue that is particularly detrimental to performance. A possible explanation for why directional alterations were so difficult may be that when participants encountered a cue that appeared in a spatially expected position, this exogenous information was taken as confirmation that the movement should be completed as expected, and no further analyses, requiring the SAS, was necessary. At the very least, it indicates that when the participants expected to move to the routine target location and the exogenous cue information was congruent with that expectancy, attention, and likely prefrontal activation, was greatly biased toward that routine location. This would make it less likely that the ventral fronto-parietal network would become engaged to interrupt the CS.

Finally, even though combined alterations also required a change from the expected movement plan and, therefore, a switch to the SAS, participants in both Experiments 1 and 2 were better able to manage this switch than when the trial involved a directional alteration. This difference in accuracy between the two types of alterations is particularly enlightening with respect to the conditions under which action slips are likely to occur.

The key feature that distinguished directional and combined alterations was that the latter involved the altered arrow cue's not only pointing to an unexpected target button, but also appearing in an unexpected spatial location. Since participants were significantly better at responding correctly to these combined alterations, it follows that there must be something about the cue's appearing in an unexpected location that facilitates an interruption of the CS, a switch to the SAS, and therefore correct action production. Specifically, whenever a change to the routine movement was required, performance was enhanced by exogenously cuing that change in a physical location that was spatially incongruent with the expected movement. In other words, cuing a movement change was particularly effective when it happened in an unexpected location as well, which possibly increased response competition between the expected target location and the correct target location. This would be consistent with a hypothesis that attention-related activation in the brain was biased away from the expected target location, via the prefrontal cortex (Miller \& Cohen, 2001), and in turn, it may have facilitated the "circuit-breaking" function of the ventral fronto-parietal network (Corbetta \& Shulman, 2002). This finding that, when required, participants were better able to change their movement goal in a routine task if that change was cued in an unexpected spatial location suggests a possible strategy for preventing slips in real life.

Another key strategy for preventing action slips comes from the timing data from these experiments. It is clear that in order to prevent a slip on the SIT, an interruption of the routine action is required. It is possible that such an interruption may be facilitated by increased activation or bias away from the expected target, which is consistent with Miller and Cohen's (2001) theory. Also consistent with Miller and Cohen, as well as Braver and Cohen (2000), is that the likelihood of such an interruption occurring is directly related to mechanisms that are extremely time sensitive. Indeed, the results of this study indicate that on an altered trial, when participants initiated a movement more quickly, they were less able to adjust their expected movement plan to coincide with an altered cue. However, simply initiating and/or moving more quickly on preceding trials did not predict the likelihood of committing a slip. To more clearly dissociate this apparent relationship between speed and error commission, it may be helpful to determine whether an error on an altered trial can be predicted from a participant's IT on a preceding trial. In such an experiment, the participant's IT would be monitored trial by trial, and when an IT was faster than a predetermined threshold, an alteration would be introduced on the subsequent trial. The extent to which an error did occur on these trials would provide insight into the effect of speeded response preparation on error production. This sort of active manipulation might better indicate the timing characteristics of when an error will occur and, thereby, the optimal task pacing for preventing action slips. 
Furthermore, when participants were able to avoid an action slip by adjusting their expected movement plan on an altered trial, our data indicate that significant increases in MT were required. Future SIT research would benefit from more sophisticated measurement tools, such as kinematic movement tracking, which might elucidate what a participant is actually doing during this MT phase. We expect that on an altered trial, participants might be committing a microslip, wherein they move toward the expected target location, before self-correcting and moving in accordance with the arrow cue's designated direction. If this were the case, it might be possible to determine the point in time at which a participant's movement trajectory changes so as to determine the time needed to make a self-correction. It may be that there is some type of temporal threshold beyond which self-correction may not be possible and an error will be made. Examining the time course of these selfcorrections may provide important insight into understanding the microstructure of action slips

In sum, the SIT provides an opportunity to study action slips within a context of a sequence of routine movements. As such, it contains an added a layer of complexity that other tasks that are commonly used to elicit errors do not have. The task also is designed to allow the examination of the timing microstructure of both the actual altered movement and the movement that precedes and follows an alteration. Furthermore, the design of the task allows for the consideration of the individual impacts of expectation, exogenous cue information, and endogenous cue information on the biasing of attention and/or activation, perhaps via the prefrontal cortex, to targets. That said, the SIT task basically creates a situation in which conflict exists between the spatial location of a cue, the pointed direction of a cue, and one's routine movement. This conflict appears to result, to differing degrees depending on the circumstances, in a failure on the part of the attention system to deploy appropriate cognitive control. It remains to be seen whether the creation of action slips with the SIT is specific to the visual modality. Certainly this could be the case; however, considering the design of the SIT future experiments investigating the effectiveness of exogenous and endogenous auditory and/or tactile cuing is warranted.

\section{Appendix}

Attention Related Cognitive Errors Scale (ARCES; Cheyne et al. 2006)

The following statements are about minor mistakes and absent-mindedness everyone notices from time to time, but we have very little information about just how common they are. The great majority of time these little foibles are harmless, though they do have serious safety implications in industry and everyday life. We want to know how frequently these sorts of things have happened to you.

There are 12 Questions. Please answer by circling a number on the scale provided below each question.

1. I have gone to the fridge to get one thing (e.g.,milk) and taken something else (e.g., juice).

\begin{tabular}{|c|c|c|c|c|}
\hline Never & Rarely & Sometimes & Often & Very often \\
\hline 1 & 2 & 3 & 4 & 5 \\
\hline \multicolumn{5}{|c|}{$\begin{array}{l}\text { 2. I go into a room to do one thing (e.g., brush my teeth) and end up doing something } \\
\text { else (e.g., brush my hair). }\end{array}$} \\
\hline Never & Rarely & Sometimes & Often & Very often \\
\hline 1 & 2 & 3 & 4 & 5 \\
\hline
\end{tabular}

3. I have lost track of a conversation because I zoned out when someone else was talking.

$\begin{array}{ccccc}\text { Never } & \text { Rarely } & \text { Sometimes } & \text { Often } & \text { Very often } \\ 1 & 2 & 3 & 4 & 5\end{array}$

4. I have absent-mindedly placed thing sin unintended locations (e.g., putting milk in the pantry or sugar in the fridge).

$\begin{array}{ccccc}\text { Never } & \text { Rarely } & \text { Sometimes } & \text { Often } & \text { Very often } \\ 1 & 2 & 3 & 4 & 5\end{array}$

5. I have gone into a room to get something, got distracted, and wondered what I went there for.

$\begin{array}{ccccc}\text { Never } & \text { Rarely } & \text { Sometimes } & \text { Often } & \text { Very often } \\ 1 & 2 & 3 & 4 & 5\end{array}$

6. I begin one task and get distracted into doing something else.

$\begin{array}{ccccc}\text { Never } & \text { Rarely } & \text { Sometimes } & \text { Often } & \text { Very often } \\ 1 & 2 & 3 & 4 & 5\end{array}$

7. When reading I find that I have read several paragraphs without being able to recall what I read.

$\begin{array}{ccccc}\text { Never } & \text { Rarely } & \text { Sometimes } & \text { Often } & \text { Very often } \\ 1 & 2 & 3 & 4 & 5\end{array}$

8. I make mistakes because I am doing one thing and thinking about another.

$\begin{array}{ccccc}\text { Never } & \text { Rarely } & \text { Sometimes } & \text { Often } & \text { Very often } \\ 1 & 2 & 3 & 4 & 5\end{array}$

9. I have absent-mindedly mixed up targets of my action (e.g., pouring or putting something into the wrong container).

$\begin{array}{ccccc}\text { Never } & \text { Rarely } & \text { Sometimes } & \text { Often } & \text { Very often } \\ 1 & 2 & 3 & 4 & 5\end{array}$

10. I have to go back to check whether I have done something or not (e.g., turning out lights, locking doors).

$\begin{array}{ccccc}\text { Never } & \text { Rarely } & \text { Sometimes } & \text { Often } & \text { Very often } \\ 1 & 2 & 3 & 4 & 5\end{array}$

11. I have absent-mindedly misplaced frequently used objects, such as keys, pens, glasses, etc.

$\begin{array}{ccccc}\text { Never } & \text { Rarely } & \text { Sometimes } & \text { Often } & \text { Very often } \\ 1 & 2 & 3 & 4 & 5\end{array}$

12. I fail to see what I am looking for even though I am looking right at it.

$\begin{array}{ccccc}\text { Never } & \text { Rarely } & \text { Sometimes } & \text { Often } & \text { Very often } \\ 1 & 2 & 3 & 4 & 5\end{array}$




\section{References}

Aron, A. R., Durston, S., Eagle, D. M., Logan, G. D., Stinear, C. M., \& Stuphorn, V. (2007). Converging evidence for a fronto-basal-ganglia network for inhibitory control of action and cognition. Journal of Neuroscience, 27, 11860-11864.

Baranowski, T. (1988). Validity and reliability of self report measures of physical activity: An information-processing perspective. Research Quarterly for Exercise and Sport, 59, 314-327.

Betsch, T., Haberstroh, S., Molter, B., \& Glockner, A. (2004). Oops, I did it again-relapse errors in routinized decision making. Organizational Behavior and Human Decision Processes, 93, 62-74.

Botvinick, M., \& Bylsma, L. M. (2005). Distraction and action slips in an everyday task: Evidence for a dynamic representation of task content. Psychonomic Bulletin \& Review, 12, 1011-1017.

Botvinick, M. M., Cohen, J. D., \& Carter, C. S. (2004). Conflict monitoring and anterior cingulate cortex: An update. Trends in Neurosciences, 8, 539-546.

Braver, T. S., Barch, D. M., Gray, J. R., Molfese, D. L., \& Snyder, A. (2001). Anterior cingulate cortex and response conflict: Effects of frequency, inhibition and errors. Cerebral Cortex, 11, 825836.

Braver, T. S., \& Cohen, J. D. (2000). On the control of control: The role of dopamine in regulating prefrontal function and working memory. In S. Monsell \& J. Driver (Eds.), Attention and performance, XVIII: Control of cognitive processes (pp. 712 737). Cambridge: MIT Press.

Broadbent, D. E., Cooper, P. F., FitzGerald, P., \& Parkes, K. P. (1982). The Cognitive Failures Questionnaire (CFQ) and its correlates. British Journal of Clinical Psychology, 21, 1-16.

Buxbaum, L. J., Schwartz, M. F., \& Montgomery, M. W. (1998). Ideational apraxia and naturalistic action. Cognitive Neuropsychology, 15, 617-643.

Carter, C. S., Braver, T. S., Barch, D. M., Botvinick, M. M., Noll, D., \& Cohen, J. D. (1998). Anterior cingulated cortex, error detection, and the online monitoring of performance. Science, 280, 747-749

Casey, B. J., Tottenham, N., \& Fossella, J. (2002). Clinical, imaging, lesion and genetic approaches toward a model of cognitive control. Developmental Psychobiology, 40, 237-254.

Chambers, C. D., Bellgrove, M. A., Stokes, M. G., Henderson, T. R., Garavan, H., Robertson, I. H., . . . Mattingly, J. B. (2006). Executive "brake failure" following deactivation of human frontal lobe. Journal of Cognitive Neuroscience, 18, 444-455

Cheyne, J. A., Carriere, J., \& Smilek, D. (2006). Absent-mindedness: Lapses of conscious awareness and everyday cognitive failures. Consciousness and Cognition, 15, 578-592.

Cheyne, J. A., Solman, G. J. F., Carriere, J. S. A., \& Smilek, D. (2009). Anatomy of an error: A bidirectional state model of task engagement/disengagement and attention-related errors. Cognition, 111, 98-113.

Corbetta, M., \& Shulman, G. L. (2002). Control of goal-directed and stimulus-driven attention in the brain. Nature Reviews Neuroscience, 3, 201-215.

Eriksen, B. A., \& Eriksen, C. W. (1974). Effects of noise letters upon the identification of a target letter in a nonsearch task. Perception \& Psychophysics, 16, 143-149.

Fassbender, C., Murphy, K., Foxe, J. J., Wylie, G. R., Javitt, D. C., Robertson, I. H., \& Garavan, H. (2004). A topography of executive functions and their interactions revealed by functional magnetic resonance imaging. Cognitive Brain Research, 20, 132-143.

Giovannetti, T., Bettcher, B. M., Brennan, L., Libon, D. J., Wambach, D., \& Seter, C. (2010). Target-related distracters disrupt object selection in everyday action: Evidence from participants with dementia. Journal of the International Neuropsychological Society, 16, 484-494.

Giovannetti, T., Schwartz, M., \& Buxbaum, L. (2007). The coffee challenge: A new method for the study of everyday action errors. Journal of Clinical and Experimental Neuropsychology, 29, 690 705.

Gratton, G., Coles, M. G. H., \& Donchin, E. (1992). Optimizing the use of information: Strategic control of activation of responses. Journal of Experimental Psychology. General, 121, 480-506.

Heckhausen, H., \& Beckmann, J. (1990). Intentional action and action slips. Psychological Review, 97, 36-48.

Humphreys, G. W., Forde, E. M. E., \& Francis, D. (2000). The organization of sequential actions. In S. Monsell \& J. Driver (Eds.), Attention and performance XVIII: Control of cognitive processes (pp. 427-472). Cambridge: MIT Press.

James, W. (1890). The principles of psychology (Vol. I). New York: Holt.

Jonides, J., Badre, D., Curtis, C., Thompson-Schill, S. L., \& Smith, E. E. (2002). Mechanisms of conflict resolution in prefrontal cortex. In D. T. Stuss \& R. T. Knight (Eds.), Principles of frontal lobe function (pp. 233-265). New York: Oxford University Press.

Jonsdottir, M. K., Adolfsdottir, S., Cortex, R. D., Gunnarsdottir, M., \& Gustafsdottir, A. H. (2007). A diary study of action slips in healthy individuals. The Clinical Neuropsychologist, 21, 875883.

Kanfer, F. H. (1970). Self-monitoring: Methodological limitations and clinical applications. Journal of Consulting and Clinical Psychology, 35, 148-150.

Kok, A., Ridderinkhof, K. R., \& Ullsperger, M. (2006). The control of attention and actions: Current research and future developments. Brain Research, 1105, 1-6.

Logan, G. D., Cowan, W. B., \& Davis, K. A. (1984). On the ability to inhibit simple and choice reaction time responses: A model and a method. Journal of Experimental Psychology. Human Perception and Performance, 10, 276-291.

Miller, E. K., \& Cohen, J. D. (2001). An integrative theory of prefrontal cortex function. Annual Review of Neuroscience, 24, 167-202.

Norman, D. A., \& Shallice, T. (1986). Attention to action: Willed and automatic control of behavior. In R. J. Davidson, G. E. Schwartz, \& D. Shapiro (Eds.), Consciousness and self-regulation: Advances in research and theory (Vol. 4, pp. 1-18). New York: Plenum.

Norman, D. A., \& Shallice, T. (2000). Attention to action: Willed and automatic control of behavior. In M. S. Gazzaniga (Ed.), Cognitive neuroscience: A reader (pp. 1-18). Malden: Blackwell.

Paxton, J. L., Barch, D. M., Racine, C. A., \& Braver, T. S. (2008) Cognitive control, goal maintenance, and prefrontal function in healthy aging. Cerebral Cortex, 18, 1010-1028.

Rabbitt, P. M. A., \& Rodgers, B. (1977). What does a man do after he makes an error? An analysis of response programming. Quarterly Journal of Experimental Psychology, 29, 727-743.

Reason, J. (1977). Skill and error in everyday life. In M. Howe (Ed.), Adult learning (pp. 21-44). London: Wiley.

Reason, J. (1979). Actions not as planned: The price of automization. In G. Underwood \& R. Stevens (Eds.), Aspects of consciousness (pp. 67-89). London: Academic Press.

Reason, J. (1984). Lapses of attention in everyday life. In R. Parasuraman \& D. R. Davies (Eds.), Varieties of attention (pp. 515-549). Orlando: Academic Press.

Reason, J., \& Mycielska, K. (1982). Absent-minded? The psychology of mental lapses and everyday error. Englewood Cliffs: PrenticeHall.

Robertson, I. H. (2003). The absent mind: Attention and error. The Psychologist, 16, 476-479.

Robertson, I. H., Manly, T., Andrade, J., Baddeley, B. T., \& Yiend, J. (1997). 'Oops!': Performance correlates of everyday attentional 
failures in traumatic brain injured and normal subjects. Neuropsychologia, 35, 747-758.

Rosvold, H. E., Mirsky, A. F., Sarason, I., Bransome, E. D., \& Beck, L. H. (1956). A continuous performance test of brain damage. Journal of Consulting Psychology, 20, 343-350.

Schwartz, M. F. (1995). Re-examining the role of executive functions in routine action production. Annals of the New York Academy of Science, 769, 321-335.

Shadish, W. R., Cook, T. D., \& Campbell, D. T. (2002). Experimental and quasi- experimental designs for generalized causal inference. Boston: Houghton Mifflin.

Shallice, T., \& Burgess, P. (1993). Supervisory control of action and thought selection. In Baddeley \& Weiskrantz (Eds.), Attention: Selection, awareness and control (pp. 171-187). Oxford: Oxford University Press.
Sharp, D. J., Bonnelle, V., De Boisserzon, X., Beckmann, C. F., James, S. G., Patel, M. C., \& Mehta, M. A. (2010). Distinct frontal systems for response inhibition, attentional capture and error processing. Proceedings of the National Academy of Sciences, 107, 6106-6111.

Simon, J., \& Berbaum, K. (1990). Effect of conflicting cues on information processing: The 'Stroop Effect' vs. The 'Simon Effect.'. Acta Psychologica, 73, 159-170.

Tipper, S. P., Lortie, C., \& Baylis, G. C. (1992). Selective reaching: Evidence for action-centered attention. Journal of Experimental Psychology. Human Perception and Performance, 18, 891-905.

Wallace, J. C., Vodanovich, S. J., \& Restino, B. M. (2003). Predicting cognitive failures from boredom proneness and daytime sleepiness scores: An investigation within military and undergraduate samples. Personality and Individual Differences, 34, 635-644. 